\title{
Yield Stress of Alcoflood and Xanthan Polymers Solutions and Their Emulsions with Crude Oil
}

\author{
Mamdouh T. Ghannam, Mohamed Y. E. Selim, Abdulrazag Y. Zekri, and Nabil Esmail
}

\begin{abstract}
Experimental investigation of yield stress measurements for Alcoflood and Xanthan polymers solutions and their emulsions with crude oil were studied by using RheoStress RS100 under controlled stress mode. The yield stress study was investigated over a wide range of polymer concentration, two concentrations of crude oil, and different type of polymers. For the same polymer concentration of $104 \mathrm{ppm}$, the aqueous solution of AF1285 reported a higher resistance to flow than the aqueous solution of AF1235. The apparent yield stresses for Alcoflood aqueous solutions and their crude oil emulsions are increased by polymer concentration. Higher concentration of Xanthan aqueous solutions showed higher ascending and descending rheograms. For Xanthan concentration of $\leq 1000 \mathrm{ppm}$, very low yield stresses are found for all the Xanthan solutions and oil emulsions. For $\mathbf{7 5 \%}$ crude oil emulsions, almost similar rheograms behaviors are reported for all the tested Xanthan concentrations. The yield stress analysis showed that the higher Xanthan concentration leads to a higher resistance for both of aqueous solutions and emulsions to startup.
\end{abstract}

Index Terms-Yield stress, Alcoflood polymer, Xanthan gums, crude oil emulsion.

\section{INTRODUCTION}

Polymer aqueous solutions can be utilized in the enhanced oil recovery to extract some of the residual crude oil (i.e. around $40-50 \%$ of the original oil) available in the oil formation which cannot be obtained during the traditional primary techniques. In the enhanced oil recovery stage, polymers are commonly used to modify the rheology of the aqueous phase to improve mobility ratio, sweep efficiency, and increase recovery and oil production rates. The process of polymer injection into the oil well usually leads to the formation of crude oil-polymer emulsion. The knowledge of the oil phase interaction within the aqueous continuous phase is necessary to understand the oil displacement by polymer solution. Also, understanding of the rheological properties of crude oil-polymer emulsion system is important in pipeline

Manuscript received November 9, 2017; revised February 10, 2018

Mamdouh T. Ghannam and Abdulrazag Y. Zekri are with Department of Chemical and Petroleum Engineering, Faculty of Engineering, United Arab Emirates University, P.O. 15551, Al-Ain, United Arab Emirates (e-mail: mamdouh.ghannam@uaeu.ac.ae).

Mohamed Y. E. Selim is with Department of Mechanical Engineering Faculty of Engineering, United Arab Emirates University, P.O. 15551, Al-Ain, United Arab Emirates.

Nabil Esmail is with Department of Mechanical Engineering, Concordia University, 1455 de Maisonneuve Boulevard W., Montreal, Quebec, Canada M3G 1M8. transportation. There are two main polymers being used in the polymer flooding stage. These polymers are polyacrylamide and polysaccharides biopolymer or Xanthan gum [1], [2].

The first type of polymer can be prepared by the polymerization of the acrylamide monomer to produce polyacrylamide. Through hydrolysis some of the acrylamide monomers are converted to carboxylate groups with a negative charge. The polyacrylamide polymer is commonly utilized in modern technologies as a thickening agent, suspending agent, turbulent reduction agent and in the enhanced oil recovery [3], [4]. The second type of polymer that can be used in the enhanced oil recovery is the Xanthan gum, which is a high molecular weight extracellular polysaccharide produced by bacteria of the genus Xanthomonas campestris. Several important applications such as food, pharmaceutical, and oil industries involve Xanthan gum in their processes [5], [6].

Numerous rheological studies on polyacrylamide aqueous solutions have been found in the literature. Among these, $\mathrm{Li}$ and McCarthy [7] studied the flow behavior of polyacrylamide aqueous solution in pipelines, Ghoniem et al. [8] and Chang and Darby [9] reported mechanical degradation of polyacrylamide aqueous solution. Dupuis et al. [10] studied the rheological behavior of polyacrylamide and glycerol aqueous solution, and they found time dependence viscosity behavior. Ait-Kadi et al. [11] investigated the role of salt on the viscoelastic behavior of the polyacrylamide solution, and they found that the salt provided a stability effect on the solution viscosity. Shin and Cho [12] investigated the temperature effect on the polyacrylamide aqueous solution, and reported that the viscosity showed temperature dependence at low shear rates while temperature independence at high shear rate.

Many biopolymers can be used in oil recovery processes, while Xanthan gum has been the most utilized biopolymer [13]. Aqueous solutions of Xanthan gum exhibit pseudoplastic behavior with very good suspending properties. Therefore, Xanthan solutions can be used as a suspending, stabilizing, thickening and emulsifying agent for food, cosmetics, pharmaceuticals and oil recovery among other applications [14]. Several experimental studies on the viscosity investigation of the Xanthan aqueous solution have been found. Some examples are: Whistler and BeMiller [15] who found strong pseudoplasticity results for the Xanthan solutions due to the formation of high molecular weight aggregates of stiff rod molecules. Milas and Rinaudo [16] reported that the Xanthan macromolecules in the ordered helical structure would stiffen the polysaccharide solution making Xanthan one of the stiffest natural biopolymers [17]. 
Several experimental studies are carried out on the rheological measurements of emulsions in which the continuous phase is Newtonian fluid [18]-[20]. For a very dilute concentration of oil phase into an aqueous phase, viscosity increases linearly with oil concentration. For a medium oil concentration, viscosity increases nonlinearly while the emulsion is still Newtonian. However, at a high oil concentration, the flow exhibits a non-Newtonian pseudoplastic profile with associated yield stress at oil concentration higher than 75\% [21]-[23]. Limited numbers of rheological investigations have been found on oil emulsion in which a non-Newtonian polymeric solution is used as a continuous phase [24]-[26]. Sosa-Herrera et al. [27] examined the rheological properties of oil emulsions prepared with 30\% sunflower oil and different sodium caseinate/gellan gum mixtures at $25{ }^{\circ} \mathrm{C}$. They reported that the emulsions without gellan were almost Newtonian behavior. However, when $0.03 \%$ or higher of gellan concentration was added into the emulsion, a non-Newtonian of shear thinning behavior was found.

Study of the yield stress behavior of the polymer aqueous solutions and crude oil emulsions is necessary for numerous industrial applications such as the oil industry for the initiation of the aqueous polymer solution or pumping an oil-polymer emulsion into a transportation pipeline. It is widely accepted that the mechanical characteristics of structured liquids change over a narrow low range of stress level. At stress below this level, the structured liquid behaves as elastic profile showing solid similar behavior. At stress above this level, the tested material displays continuous deformation showing viscous liquid behavior. This stress level is called the apparent yield stress [28].

This study will focus on the apparent yield stress comparison for the two most widely utilized commercial polymers and their emulsions with crude oil using a controlled-stress rheometer. These are Alcoflood polymer (i.e. polyacrylamide) and Xanthan gums (i.e. polysaccharides biopolymer). This experimental study examined a wide range of crude oil, Alcoflood, and Xanthan gums concentrations.

\section{EXPERIMENTAL}

One of the important applications for the Alcoflood and Xanthan polymers is in the enhanced oil recovery stage either to recover some of the remaining oil in the formation or to push the surfactant solutions in the tertiary production stage. The objective of this investigation is to study the yield stress comparison of different aqueous solutions of Alcoflood and Xanthan gums and their emulsions with crude oil using Rheostress RS100 rheometer. The utilizing of controlled stress rheometer in CS-mode has allowed the accurate measurements of yield stress in comparison with controlled rate technique. Therefore, the investigation of yield stress at low shear rate of non-Newtonian profile is possible utilizing CS-mode [29]. This study examined a wide range of polymer concentrations of 0-104 ppm, two crude oil concentrations of $25 \& 75$ volume \%, and different types of polymers. Crude oil-polymer emulsions were prepared from crude oil, polymer aqueous solution, and surfactant material.

Triton X-100 was used as an emulsifying agent to prepare crude oil-polymer emulsion for yield stress measurements. Polymer aqueous solution was prepared first by complete dissolving of polymer into distilled water. Then the emulsion was prepared by gradual mixing of crude oil into the prepared aqueous solution that contained $1 \%$ by volume of Triton $\mathrm{X}-100$. The presence of an emulsifier during the preparation of crude oil-polymer emulsion is necessary to achieve strong repulsion mechanism between the droplets of the crude oil phase. Therefore, the emulsifier role is to avoid the coalescence mechanism between droplets phase and thus stabilizes the emulsion for a longer time.

\section{A. Crude Oil}

A North Sea crude oil delivered by Shell Canada Limited was employed in all experimental measurements. The crude oil viscosity equals $7.16 \mathrm{mPas}$ at $40{ }^{\circ} \mathrm{C}$, and density is 880.6 $\mathrm{kg} / \mathrm{m} 3$ at $15{ }^{\circ} \mathrm{C}$.

\section{B. Surfactant Material}

Triton X100 from Sigma-Aldrich Canada Ltd. with a specific gravity of 1.07 was used as a surfactant. A surfactant material was necessary to prepare the crude oil emulsions. In general, a surfactant material is usually added into oil-aqueous phase system as an emulsifying agent to decrease the oil-aqueous solution interfacial tension and to stabilize the presence of the oil droplets phase within the aqueous continuous phase [30].

\section{Alcoflood Polymers}

Two Alcoflood polymers of AF1235 and AF1285, from Ciba Specialty Chemicals (Bradford, West Yorks, England), were examined in this study. Alcoflood materials are high molecular weight polyacrylamide copolymers. AF1235 is recommended for low-medium permeability reservoirs, whereas AF1285 is applied for high permeability reservoirs. The Alcoflood bulk density is $800 \mathrm{~kg} / \mathrm{m3}$ with intrinsic viscosity equal to 12 and 24 for AF1235 and AF1285, respectively. The water-soluble Alcoflood materials were supplied in a white granular powder form. The Alcoflood aqueous solutions were prepared by adding a certain weight of polymer material to 0.25 liter of warm distilled water. Enough time was given to achieve complete polymer dissolution without external mixing to avoid any mechanical degradation on the polymer network.

\section{Xanthan Gums}

Two Xanthan gums were examined for this study, the first gum was a chemical grade from Sigma-Aldrich Canada Ltd (Oakville, Ontario L6H 6J8, Canada) with product \# G1253 under the product name of Sigma. The second gum was an industrial grade of Xanthan gum from CP Kelco (Atlanta-GA 30339, USA) with product \# 10040282 and product name Kelzan. Both gums are white to tan colored powders and used in non-food applications such as a thickener and rheology control agents. The Xanthan solutions were prepared by 
mixing the powder slowly in 0.25 liter of warm distilled water to reach the required concentration. The solutions were gently stirred until all the Xanthan gum completely dissolved. Since Xanthan solution is biodegradable, $1.0 \mathrm{gm}$ formaldehyde was added and the solutions were stored at $4 \mathrm{oC}$ until use to avoid bacterial growth.

\section{E. RS100 Rheometer}

The yield stress measurements of aqueous solutions and crude oil-polymers emulsions were carried out at a room temperature of 22 oC using RheoStress RS100 under controlled stress mode. A water bath was connected to the rheometer to control the applied temperature of the RS100 system. The controlled stress mode allows the most direct technique for the yield stress measurements. The applied shear stress on the examined sample is gradually increased without shear flow reported till the yield stress is reached. The shear stress-shear rate data were collected using cone-plate sensor with a cone angle of 4o. The cone diameter was $35 \mathrm{~mm}$ with a gap of $0.137 \mathrm{~mm}$ at the cone tip.

\section{Results AND DisCUSSION}

Some of the enhanced oil recovery's polymer solutions and their crude oil emulsions are subjected to different stages of storage, pumping, and pipeline transportations; therefore it is necessary to study the apparent yield stress of these aqueous solutions and their emulsions in the presence of Alcoflood and Xanthan gums. Utilizing control stress mode of the RS100 allows measuring very low yield stress. To establish the rheogram behavior of polymer solutions and crude oil emulsions, every sample will be subjected to two cycles of shear rate-shear stress. Up to $5000 \mathrm{ppm}$ of polymer concentration, the stress was covered over the range $0-5 \mathrm{~Pa}$ to obtain 20 points of shear rate-shear stress measurements within the period of 180 seconds to establish the upward curve (i.e. the first cycle). To construct the downward curve, i.e. the second cycle, the assigned stress was driven backward from 5 Pa to no stress applied to collect another 20 experimental points of shear rate-shear stress within 180 seconds. For the polymer concentration of $\geq 5000 \mathrm{ppm}$, the stress cycle was covered over the range up to $20 \mathrm{~Pa}$.

\section{A. Yield Stress Behavior for Alcoflood Aqueous Solutions and Oil Emulsions}

Fig. 1 displays the relationship between the assigned shear stress and the response shear rate for the aqueous solutions of the two Alcoflood tested polymers with concentrations of 104 ppm. Fig. 1 displays the upward-cycle and the downward-cycle curves established by ascending and descending the applied shear stress, respectively. The maximum assigned shear stress is $20 \mathrm{~Pa}$ as shown in Fig. 1. For the AF1235 aqueous solution of 104 ppm, Fig. 1 shows that the two curves are almost similar to each other with a slight difference between them. However, for the aqueous solution of $104 \mathrm{ppm}$ of AF1285, the upward-cycle curve is positioned slightly higher than the downward-cycle curve.
This can be referred to the flow units of the Alcoflood solutions during the descending cycle having limited time to develop to their equilibrium size corresponding to the ascending curve. Further, Fig. 1 shows that higher cycles of ascending and descending curves are reported for the aqueous solution of AF1285 than the AF1235 solution for the same concentration of $104 \mathrm{ppm}$, which results in a higher resistance to flow.

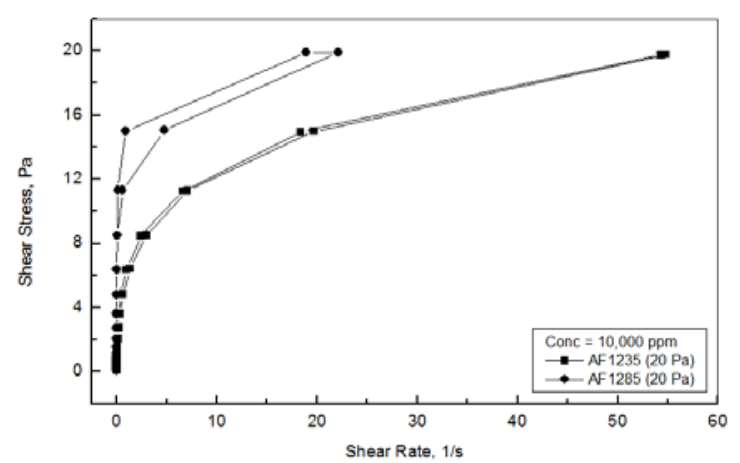

Fig. 1. Rheogram behavior for AF1235 and AF1285 aqueous solution.

Fig. 2 shows a typical example for the crude oil-Alcoflood emulsions rheograms behavior in the presence of AF1285. Fig. 2 displays the effect of different concentration of Af1285 over the range of 500-104 ppm for the oil concentration of $75 \%$. Fig. 2 reports that the full cycle of up- and down-curves increases with polymer concentration and consequently the emulsion initial flow resistance improves with polymer concentration. In a previous investigation of flow behavior of crude oil-Alcoflood polymer emulsions [26], it has been found that the well-known Casson model, equation (1), very sufficiently fits the flow behavior of the crude oil-Alcoflood polymers emulsions.

$$
\tau=\left(\tau_{\mathrm{o}}^{0.5}+\left(\dot{\gamma} \eta_{\mathrm{c}}\right)^{0.5}\right)^{2}
$$

where $\tau o$ is the apparent yield stress parameter determined by Casson model in $\mathrm{Pa}, \eta \mathrm{c}$ is the Casson apparent viscosity in Pa.s, $\tau$ is the applied shear stress in $\mathrm{Pa}$, and $\dot{\gamma}$ is the corresponding shear rate in s-1. The modeling analysis is carried out for all the examined Alcoflood aqueous solutions and their emulsions with crude oil using the Casson model of equation (1).

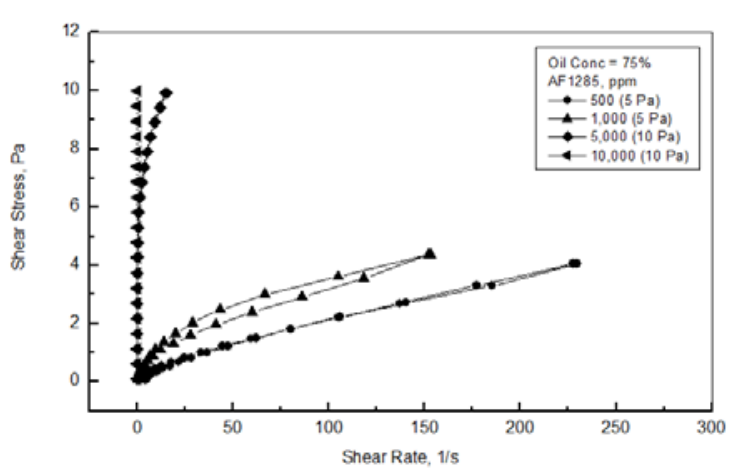

Fig. 2. Effect of AF1285 concentration on $75 \%$ oil emulsions.

The complete results of the yield stress analysis are displayed in Fig. 3. As can be noticed from Fig. 3, the yield 
stress for the Alcoflood aqueous solutions slightly increases with polymer concentration up to $103 \mathrm{ppm}$. Beyond this concentration, the yield stress strongly increases with polymer concentration. The same behavior is reported for all tested aqueous solutions and crude oil emulsions of Alcoflood polymers. Under no shear conditions, three dimensional network structures will be formed within the aqueous solution of Alcoflood polymer which results to a yield stress behavior. When the applied shear stress exceeds the yield stress, the three-dimensional network structures deformed or even break down. The strength level of the three-dimensional network structures enhances gradually with polymer concentration. Fig. 3 shows that the aqueous solutions of AF1285 provide higher yield stress values than the AF1235. Fig. 3 shows as well the comparison between the yield stress reported values of the crude oil emulsions with Af1235 \& AF1285 for the two examined oil concentrations over a wide range of polymer concentration of 0-104 ppm. As expected, both concentrations of polymer and crude oil play important role in dictating the value of the apparent yield stress. Up to polymer concentration of $103 \mathrm{ppm}$, the yield stress behavior will not significantly be affected by Alcoflood type, and both concentrations of polymer and crude oil. However, the effect of the three controlled factors of Alcoflood type and both concentrations of polymer and crude oil will be more pronounced over the polymer concentration range of 103-104 ppm. For polymer concentration > 103 ppm, AF1285 aqueous solutions cause higher apparent yield stress than aqueous solutions of AF1235. The apparent yield stresses of the Alcoflood solutions gradually decrease with further addition of crude oil.

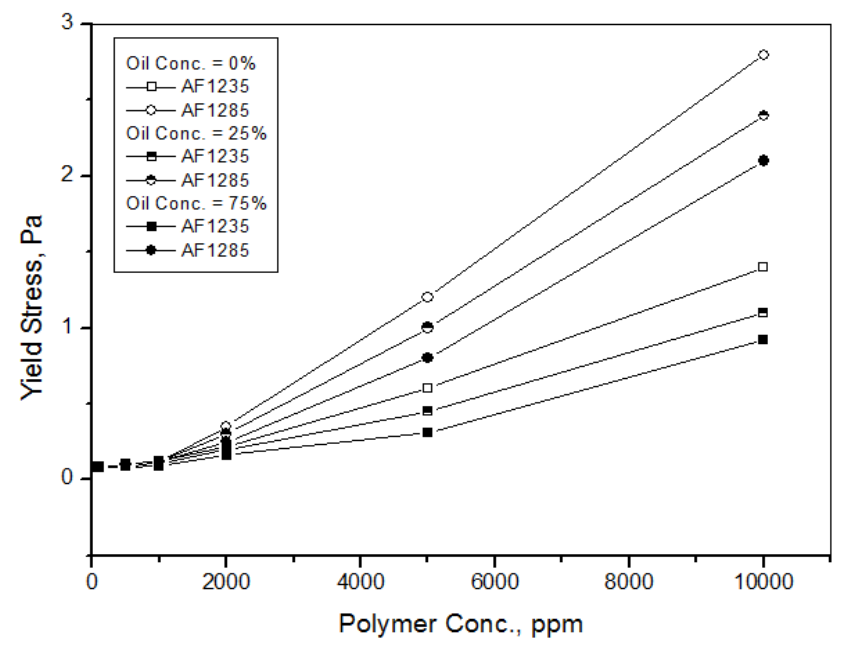

Fig. 3. Yield stress for different concerntrations of Alcoflood oil emulsions.

\section{B. Yield Stress Behavior for Xanthan Gums Aqueous Solutions and Oil Emulsions}

Fig. 4 shows the rheogram flow behavior for different concentrations of kelzan solutions as a typical example for the Xanthan gums aqueous solutions behavior. Fig. 4 displays the upward-cycle and the downward-cycle curves established by ascending and descending the applied shear stress, respectively. For the same concentration, the upward-cycle curve is positioned significantly higher than the downward-cycle curve. Higher Kelzan concentration leads to higher cycles of ascending and descending curves as can be concluded from Fig. 4, which results to higher resistance to flow. Similar behavior is reported for the Sigma aqueous solutions with different concentrations.

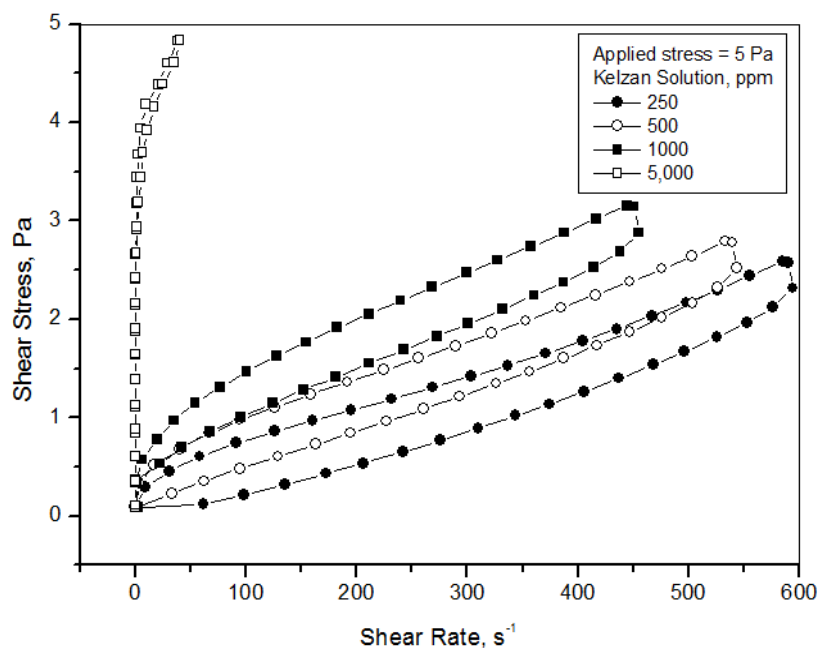

Fig. 4. Rheogram behavior for different Kelzan solutions.

Fig. 5-a shows the rheogram behavior of the $50 \%$ crude oil emulsions in the presence of different Sigma concentrations as a typical example for other emulsions with different concentrations. Fig. 5-a depicts that the ascending and descending cycles increase by Sigma concentration over the range of 250 to $5000 \mathrm{ppm}$ resulting higher yield stresses. Thus, the resistance of the initial flow of the crude oil-Xanthan emulsions will be increased with Xanthan concentration. Fig. 5-b displays the effect of different crude oil concentration in the presence of 104 ppm of Kelzan. This Fig. also shows a significant result for the further addition of crude oil into the emulsion behavior, the more oil is added to the emulsion, the higher ascending and descending cycles are reported. Fig. 5-a shows that for lower Xanthan concentration of $\leq 1000 \mathrm{ppm}$, very low yield stresses are reported for all the crude oil emulsions. However, for the higher Xanthan concentration of higher than 1000 ppm, Figs. 5-a and 5-b display much higher yield stresses. The modeling analysis employing Casson model of equation (1) was carried out to determine the yield stress for each crude oil-Xanthan emulsion.

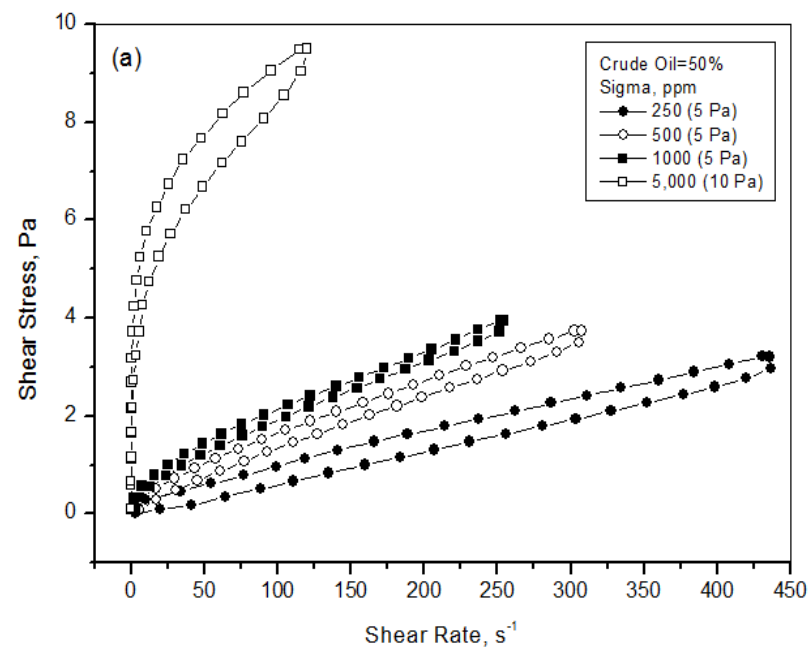




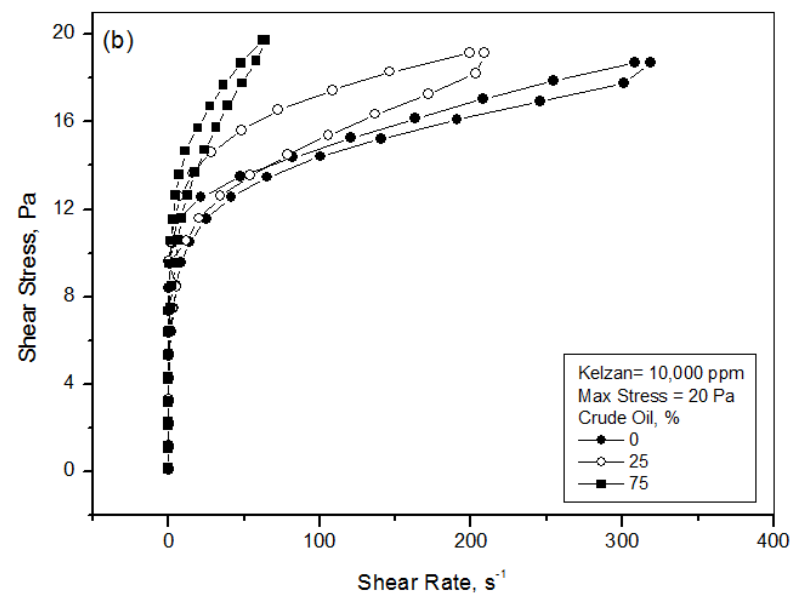

Fig. 5. Rheogram behavior for different Sigma and Kelzan emulsions.

Figs. 6-a and 6-b are typical examples for a comparison study between the rheograms behavior of Sigma- and Kelzan-crude oil emulsions. Different concentrations of Sigma and Kelzan Xanthan materials are examined in the presence of $75 \%$ crude oil. For the high crude oil concentration of 75\%, both emulsions of Sigma and Kelzan show almost similar rheograms behaviors for all tested Xanthan concentrations.
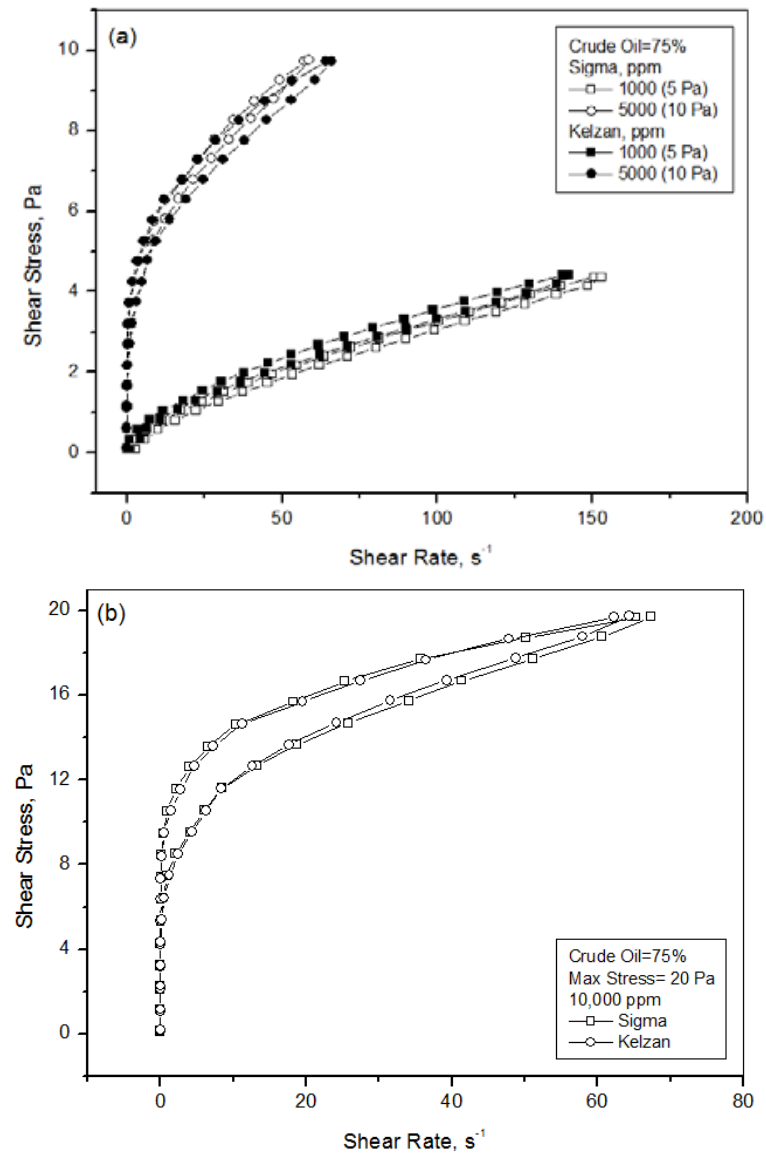

Fig. 6. Comprasion between different Sigma and Kelzan emulsions.

Modeling analysis using the Casson model of equation (1) was carried out for all the examined Xanthan aqueous solutions and their crude oil emulsions. The results in terms of yield stress are displayed in Fig. 7 versus Xanthan concentration. Fig. 7 shows that the Xanthan concentration has a strong role on the value of the apparent yield stress. Fig.
7 shows that the apparent yield stress of the Sigma solutions increases slightly up to $1000 \mathrm{ppm}$ and strongly increases with concentration over the range of 103-104 ppm. Fig. 7 displays a similar behavior for the Kelzan solutions with lower values of the apparent yield stresses. In addition, Fig. 7 shows the apparent yield stresses for Xanthan-75\% crude oil emulsions for Sigma and Kelzan. As can be found from Fig. 7, both types of Xanthan emulsions reported lower yield stresses in comparison with their aqueous solutions. Addition more Xanthan gums into the aqueous solutions raises the apparent yield stresses which leads to a higher resistance of the initial flow. However, the addition of crude oil into the aqueous solutions lowers the value of the apparent yield stresses. This can be attributed to the slight negative behavior of the oil presence on the network structure of the Xanthan material that formed within the aqueous solution under no shear status.

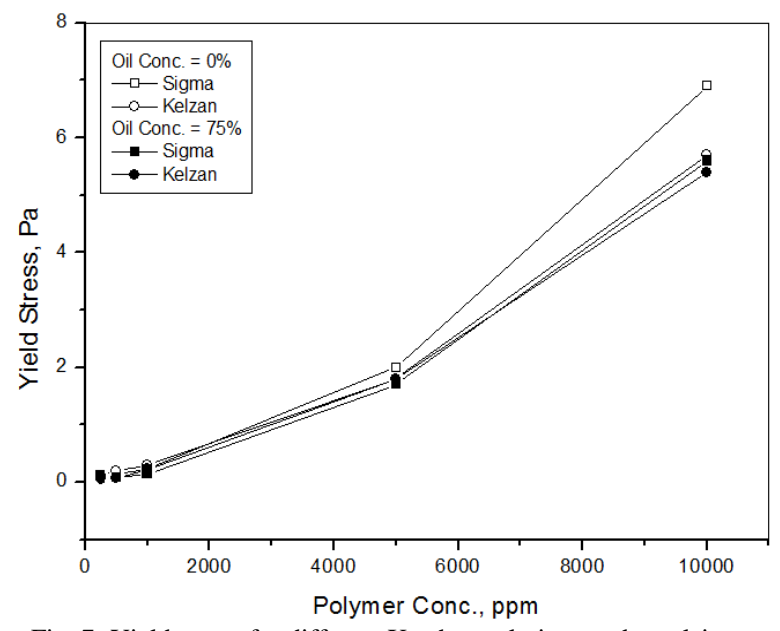

Fig. 7. Yield stress for different Xanthan solutions and emulsions.

\section{Comparison between Alcoflood and Xanthan Yield Stress Behaviors}

The detailed discussion of the rheogram behaviors for the Alcoflood and Xanthan aqueous solutions and their emulsions with crude oil are presented in the previous sections. It is useful for the current work to study the similarities and differences in the rheogram behaviors and yield stress measurements of the tested Alcoflood and Xanthan solutions and their emulsions.

Figs. 8-a and 8-b display the effect of the Alcoflood and the Xanthan gums concentration on their aqueous solutions rheogram behaviors. Low and high concentrations of Alcoflood and Xanthan gum solutions were tested in each case. For low concentration of 103 ppm, Fig. 8-a shows that the rheogram behavior of the Af1235 solution is slightly higher than the corresponding behavior of the Sigma solution which is diminished with concentration till 5000 ppm. Fig. 8-b displays rheogram behaviors for AF1285 and Kelzan aqueous solutions. For low concentration of 103 ppm, Fig. 8-b shows a similar behavior to Fig. 8-a. However, for the higher concentration of $104 \mathrm{ppm}$, the two aqueous solutions coincide on top of each other till the beginning of the viscous region in which the AF1285 provides higher response than the Kelzan solution. 

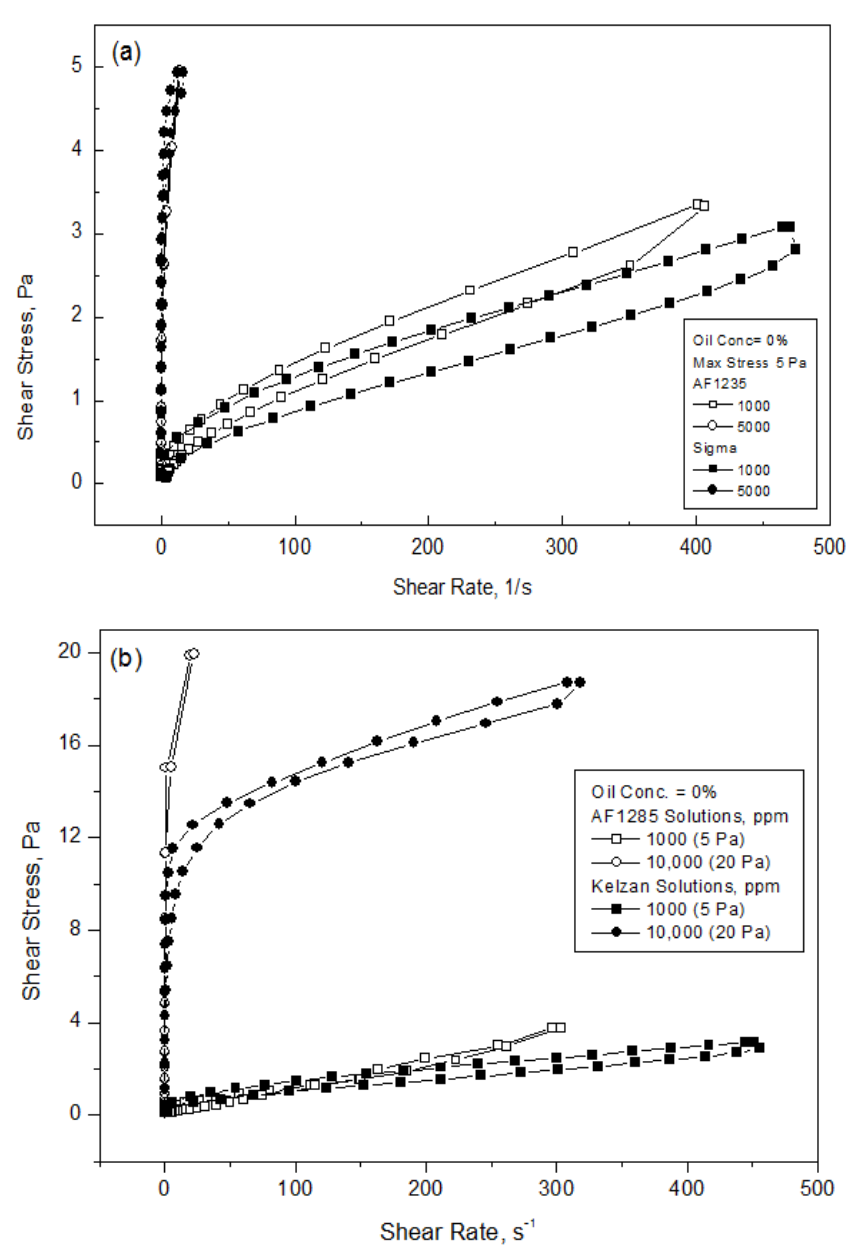

Fig. 8. Comparison between Alcoflood and Xanthan solutions.

Figs. 9-a shows the effect of different Alcoflood and Xanthan concentrations on the rheogram emulsions behavior in the presence of $25 \%$ crude oil. Fig. 9-a exhibits that the ascending and descending cycles increase by polymer concentration over the examined concentration leading to a higher yield stresses. In addition, as can be concluded from Fig. 9-a, the rheograms behaviors of the Alcoflood emulsions are higher than the corresponding Xanthan emulsions. Fig. 9-b, as a typical example, displays the effect of the crude oil addition in the presence of Alcoflood and Xanthan aqueous solutions. In general, the addition of crude oil into the aqueous phase of polymer solutions results in a significant response. Fig. 9-b shows that the more oil is added to the emulsion, the higher the ascending and descending curves are found.

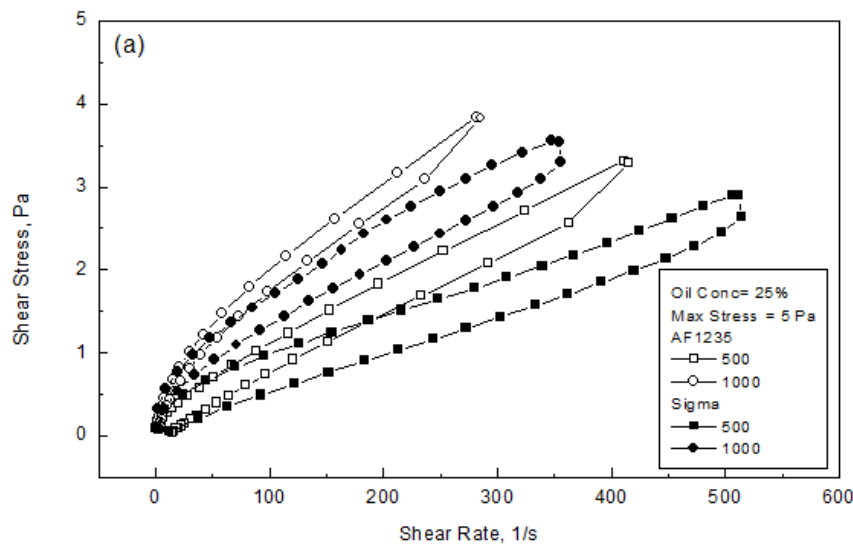

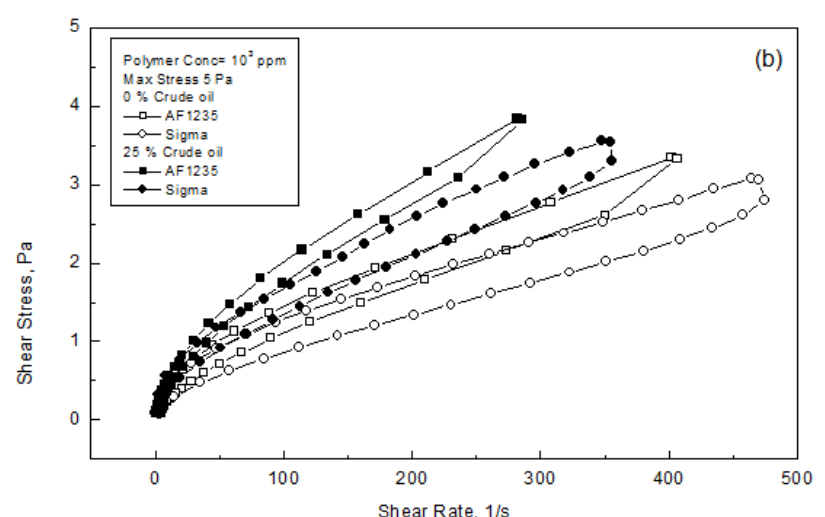

Fig. 9. Comparison between AF1235-Sigma -25\%oil emulsions.

The modeling analysis was carried out using equation (1) to determine the apparent yield stress for all the tested polymer aqueous solutions and their emulsions with crude oil. The results of this analysis are displayed in Fig. 10. Both Figs. 10-a and 10-b exhibit that the more addition of Alcoflood and Xanthan materials into the aqueous solutions enhances the yield stress which leads to a higher resistance of the initial flow. Figs. 10-a and 10-b also show that the Xanthan aqueous solutions provide significantly higher yield stresses than the Alcoflood solutions. As can be found in Figs. 10-a and 10-b, due to the addition of crude oil into the aqueous solution, the crude oil slightly lowers the value of the apparent yield stress. Also, the modeling analysis shows that the addition of the crude oil increases the Casson apparent viscosity as can be concluded from Fig. 9-b. This can be attributed to the presence of the crude oil droplets within the emulsion, which enhances the droplets interaction and therefore the Casson apparent viscosity increases with crude oil concentration.
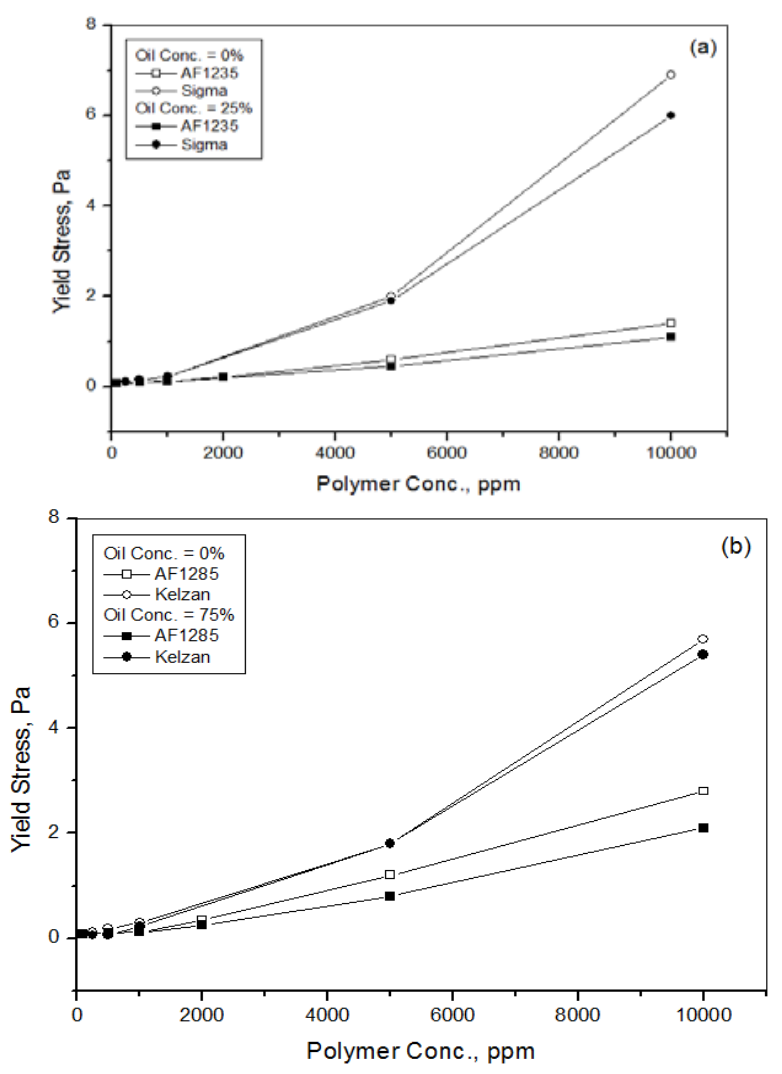

Fig. 10. Yield stresses of Alcoflood and Xanthan solutions. 


\section{CONCLUSIONS}

The yield stress investigation of Alcoflood and Xanthan aqueous solutions and their emulsions with crude oil was studied. For the same polymer concentration of 104 ppm, AF1285 aqueous solution provided higher upward and downward cycles than the aqueous solution of AF1235. Alcoflood polymer concentration enhances the initial flow resistance for both polymer aqueous solutions and their emulsions with crude oil. For polymer concentration higher than 1000 ppm, the Alcoflood polymer of AF1285 provides higher yield stress values than AF1235. Higher concentration of Xanthan solutions displayed higher cycles of upward and downward rheogram cycles. For Xanthan concentration of higher than 1000 ppm, significant yield stresses can be anticipated for the Xanthan solutions and their oil emulsions. For $75 \%$ crude oil emulsion, all concentrations of Sigma and Kelzan displayed almost similar rheograms behaviors. The yield stress of Sigma and Kelzan solutions increases slightly with polymer concentration up to 103 ppm. For concentration of higher than 1000 ppm, the yield stress raises significantly with polymer concentration. For Xanthan crude oil emulsions, the presence of crude oil leads to a slight reduction of the apparent yield stress. Aqueous solutions of Alcoflood polymer exhibit slightly higher rheograms behavior than the Xanthan solutions. The presence of Alcoflood within crude oil emulsions leads to a higher rheograms behavior than the crude oil-Xanthan emulsions. Higher ascending and descending curves resulted by adding crude oil into their aqueous solutions. The yield stress value significantly enhances with polymer concentration. For both aqueous solution and crude oil emulsion, the presence of Xanthan causes higher yield stress than the Alcoflood. The crude oil slightly decreases the value of the apparent yield stress for both Alcoflood and Xanthan emulsions.

\section{REFERENCES}

[1] A. L. Kjøniksen, N. Beheshti, H. K. Kotlar, K. Zhu, and B. Nystrom, "Modified polysaccharides for use in enhanced oil recovery applications,” European Polymer J., vol. 44, pp. 959-967, 2008.

[2] A. Samanta, A. Bera, K. Ojha, and A. Mandal, "Effects of alkali, salts, and surfactant on rheological behavior of partially hydrolyzed polyacrylamide solutions,” J. Chem. Eng. Data, vol. 55, pp. 4315-4322, 2010.

[3] F. Durst, R. Haas, and B. Kaczmar, "Flows of dilute hydrolyzed polyacrylamide solutions in porous media under various solvent conditions,” J. AppL. Polym. Sci., vol. 26, pp. 3125-3149, 1981.

[4] S. Flew and R. Sellin, "Non-Newtonian flow in porous-media-a laboratory study of polyacrylamide solutions,” J. Non-Newt. Fluid Mech., pp. 47, pp. 169-210, 1993.

[5] K. S. Kang and D. J. Pettitt, Xanthan, Gellan, Wellan, and Rhamsan in Industrial Gums, 3rd ed. R. L. Whistler, J. N. DeMiller, Academic Press: San Diego, 1993.

[6] R. A. Speers and M. A. Tung, "Concentration and temperature dependence of flow behavior of Xanthan gum dispersions,” J. Food Sci., vol. 51, pp. 96-98, 1986.

[7] T. Li and K. McCarthy, "Pipe flow of aqueous polyacrylamide solutions studied by means of nuclear magnetic resonance imaging,” J. Non-Newt. Fluid Mech., vol. 57, pp. 155-175, 1995.

[8] S. Ghoniem, G. Chauveteau, M. Moan, and C. Wolff, "Mechanical degradation of semi-dilute polymer solutions in laminar flows," Can. J. Chem. Eng., vol. 59, pp. 450-454, 1981.

[9] H. Chang and R. Darby, "Effect of shear degradation on the rheological properties of dilute drag-reducing polymer-solutions,” J. Rheol., vol. 27, pp. 77-88, 1983.
[10] D. Dupuis, F. Lewandowski, P. Steiert, and C. Wolff, "Shear thickening and time-dependent phenomena-the case of polyacrylamide solutions," $J$. Non-Newt. Fluid Mech., vol. 54, pp. 11-32, 1994.

[11] A. Ait-Kadi, P. Carreau, and G. Chauveteau, "Rheological properties of partially hydrolyzed polyacrylamide solutions,” J. Rheol., vol. 31, pp. 537-561, 1987.

[12] S. Shin and Y. Cho, "Temperature effect on the non-Newtonian viscosity of an aqueous polyacrylamide solution,” Int. Comm. Heat Mass Transfer, vol. 20, pp. 831-844, 1993.

[13] R. G. Ryles, "Chemical stability limits of water-soluble polymers used in oil recovery processes,” SPE Res. Eng., vol. 3, pp. 23-34, 1988.

[14] I. W. Sutherland, Extracellular Polysaccharides, H. J. Rehm, and G. Reed, ed., vol. 6; Biotechnology, VCH: Weinheim. 1996.

[15] R. L. Whistler and J. N. BeMiller, Carbohydrate Chemistry for Food Scientists, Eagan Press: St. Paul, MN. 1997.

[16] M. Milas and M. Rinaudo, "Conformation investigation of the bacterial polysaccharide Xanthan,” Carbohydrate Research, vol. 76, pp. 189-196, 1979.

[17] T. Coviello, K. Kajiwara, W. Burchard, M. Dentini, and V. Crescenzi, "Solution properties of Xanthan 1. Dynamic and static light scattering from native and modified Xanthans in dilute solutions," Macromolecules, vol. 19, pp. 2826-2831, 1986.

[18] P. Sherman, Industrial Rheology, Academic Press: London, 1970.

[19] T. H. Plegue, S. Frank, D. Fruman, and J. L. Zakin, "Viscosity and colloidal properties of concentrated crude oil in water emulsions," $J$. Colloid Interface Science, vol. 114, pp. 88-105, 1986.

[20] R. Pal and E. Rhodes, "Viscosity/concentration relationships for emulsions,” J. Rheol., vol. 33, pp. 1021-1045, 1989.

[21] H. Princen, "Rheology of foams and highly concentrated emulsions. II. Elastic properties and yield stress of a cylindrical model system,” $J$. Colloid Interface Science, vol. 91, pp. 160-175, 1983.

[22] H. Princen, "Rheology of foams and highly concentrated emulsions. II. Experimental study of the yield stress and wall effects for concentrated oil-in-water emulsions,” J. Colloid Interface Science, vol. 105, pp. 150-171, 1985.

[23] H. Princen and A. Kiss, "Rheology of foams and highly concentrated emulsions. III. Static shear modulus,” J. Colloid Interface Science, vol. 112, pp. 427-437, 1986.

[24] C. Han, and R. King, "Measurements of the rheological properties of concentrated emulsions,” J. Rheol. 24, 213-237, 1980.

[25] R. Pal, "Rheology of polymer-thickened emulsions,” J. Rheol., vol. 36, pp. 1245-1259, 1992.

[26] M. Ghannam, "Emulsion flow behavior of crude oil-Alcoflood polymers,” J. Chem. Eng. Japan, vol. 36, pp. 35-44, 2003.

[27] M. G. Sosa-Herrera, C. L. A. Berli, and L. P. Martinez-Padilla, "Physicochemical and rheological properties of oil-in-water emulsions prepared with sodium caseinate/gellan gum mixtures," Food Hydrocolloids, vol. 22, pp. 934-942, 2008.

[28] C. W. Macosko, Rheology-Principles, Measurements, and Applications; VCH: New York, 1994.

[29] G. Schramm, A Practical Approach to Rheology and Rheometry. Gebrueder Haake, GmbH: Karlsruhe, Germany, 1994.

[30] P. Sherman, Encyclopedia of a Emulsion Technology, P. Becher ed., vol. 1, Dekker: New York. 1983

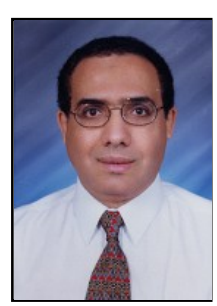

Mamdouh Ghannam is a professor of chemical engineering at University of the United Arab Emirates. He received his B.Sc. \& M.Sc. from the Cairo University, Egypt and Ph.D from University of Saskatchewan, Canada. He is a very active researcher working within several attractive fields of engineering applications such as coating and wetting behaviour of Newtonian \& non-Newtonian solutions on different solid substrate geometry, flow behaviour of crude oil- polymer emulsions, rheological characterisation of coating colour and polymer solutions, and interfacial properties of crude oil-aqueous solutions of different materials. Ghannam has authored and co-authored over 55 refereed journal publications.

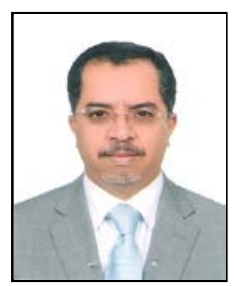

Mohamed Y. E. Selim is a professor of combustion and heat engines. He graduated from the University of Cambridge in 1992. His main area of research is combustion in internal combustion engines and other systems. He is interested on alternative fuels including biofuels and new gaseous fuels. His objectives are to reduce exhaust emissions and noise from combustion systems as well as searching for new fuels for the future. His main consultation area is the design of air conditioning and other mechanical systems for buildings. 


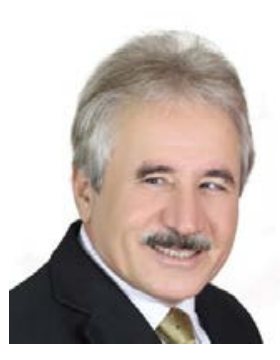

A. Y. Zekri received his B.Sc., M.S., and Ph.D degrees from the University of Southern California. He has spent more than two decades in the petroleum industry. Professor Zekri worked as a consultant to the management committees of Waha Oil Co., and Agip Oil Company. He has authored and/or co-authored more than 90 papers on new developments and technical issues in the areas of improved oil recovery, flow through porous media, and environmental aspects of petroleum production, petroleum contracts, and Enhanced Oil Recovery. He has edited and refereed technical papers in widely respected journals. Prof. Zekri has completed a number of research projects in the area of IOR/EOR to UAE and International Petroleum Industries. Professor Zekri is currently working as coordinator of oil and gas technologies, Emirates Center for energy and environment research and professor of petroleum engineering at the United Arab Emirates University.

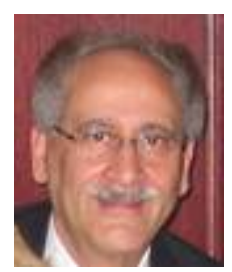

Nabil Esmail is a professor of mechanical engineering at Concordia University of Montreal, Canada, former dean of engineering in 1997-2008 at Concordia University. Professor emeritus of the University of Saskatchewan, Canada, and he is former chair of chemical engineering in 1982-1994. Esmail is a fellow of the Canadian Academy of Engineering, a fellow of the Chemical Institute of Canada, a fellow of the Engineering Institute of Canada, and a fellow of the Canadian society of mechanical engineering. Esmail has authored and co-authored over 150 refereed journal publications, to his credit the translation of three scientific books. 